\title{
Thermal activation of ordinary Portland cement-slag mortars
}

\author{
Fathollah Sajedi , Hashim Abdul Razak
}

Temperature variation caused by the heat of hydration, in mass concrete or the change of external environment, has a large influence on the mechanical properties of early-age concrete. Mechanical properties, such as compressive strength, are factors to be considered in the design and construction of concrete structures. Therefore, effects of temperature and aging, on the mechanical properties, should be studied and quantified.

According to the experimental results, concrete subjected to high temperatures at early ages, attains a higher early-age compressive and splitting tensile strength but lower later age compressive and splitting tensile strength than concretes subjected to normal temperatures [1]. Mortar and concrete are the most important elements of infrastructures and, if well-designed, they can be durable construction materials. One effective way to reduce the environmental impact is to use mineral admixtures, as a partial cement replacement. This strategy has the potential to reduce costs, conserve energy, and reduce waste volumes. Mineral admixtures are silica-based materials, such as ground granulated blast furnace slag (GGBFS), fly ash and silica fume. Mineral admixtures have been used more and more for concrete because of their strength and durability [2]. The presence of some mineral admixtures, such as GGBFS in the cement, can modify the kinetics of hydration, reduce the heat evolution and produce additional calcium silicate hydrates $(\mathrm{CSH})$ gel. These admixtures result in a noticeable performance increase to the concrete, in hot climates, in which the negative effect of the temperature is partly reduced by the pozzolanic reaction, their weak hydration heat and their great activation energies.

Several researchers reported that a high temperature improves strengths at early ages [1-3]. At a later age, the important number of formed hydrates had no time to arrange suitably, and this engendered a loss of ultimate strengths; this behavior had been called the crossover effect [4]. For ordinary Portland cement (OPC), it appears that the ultimate strength decreases, nearly linearly, with curing temperature [5]. Since GGBFS itself is nothing more than a latent hydraulic binder, it must be activated to react and provide the desirable mechanical properties. One of these activation methods 
is the thermal method [6]. The objective of this study is to produce

a data inventory of the early-age behavior, of some mechanical

properties, such as compressive strength of mortars with temperature.

In addition, to investigate the relationship between compressive

strength with temperature, and the relationship between the

compressive strength of specimens, cured in air and water, at 3

and 7 days, for $40 \%$ and $50 \%$ levels of slag replacement.

\section{Research significance}

It is known that a lot of slag is produced in the steel-iron industry

every year, throughout the entire world. If a means of consumption

for these materials is found, it would help in terms of

being environment friendly and also provide significant economic

benefits. Moreover, several researches have shown that the use

of the replacement materials in mortars and concretes has improved

durability, which has vital significance for the structures

built in aggressive environments, such as those in marine structures,

big tunnels, and bridges with long life spans. However, there

is a problem in using the materials; initial hydration is lower than

that for OPC, and then the mortars and concretes have low early-strengths. Hence, there are several ways of resolving this

problem; one of the most common methods is using thermal activation,

which is precisely the main purpose of this study.

\section{Experimental procedure}

\subsection{Mix proportions and curing}

Table 1 represents the mix designs for different mortars. In all mixes $W / B=0.33, S / B=2.25$. Silica sand was used in the mixes.

At first, based on Particle size analysis (PSA), five groups of silica

sands were mixed. One minute after that, cement and replacement-

slag were put into the mixture, followed by $1 \mathrm{~min}$ of mixing.

Mixing water was then added to the mix, and mixing was continued

for $2 \mathrm{~min}$, after which the required amount of super plasticizer

(SP) was added. Mixing was continued for 2 min before

finally, filling the moulds with fresh mortar at two layers. Each

layer was compacted with 10 impacts by a rod with $16 \mathrm{~mm}$ diameter.

$24 \mathrm{~h}$ after casting, the specimens were demoulded and heated

in water at $60 \_$, for the required time, as mentioned in Table 1, and then cured in air room temperature $\left(27 \pm 3 \_C\right)$ with $65 \pm 18 \%$

relative humidity $(\mathrm{RH})$ and water with $23 \pm 3 \_\mathrm{C}$ until the test day. 
Full text available at :

http://www.sciencedirect.com/science/article/pii/S0261306910002220

http://ac.els-cdn.com/S0261306910002220/1-s2.0-S0261306910002220-main.pdf?_tid=4b495f20-7820-11e3-

$\underline{8 d e 5-00000 a a c b 361 \& a c d n a t ~}=138915676449 f 8 a 0 a 270 b 4 c 08 c 72 a 8 f 2 a f d c 01 e 44 d$ 